Article

\title{
Global Optimization for Mixed-Discrete Structural Design
}

\author{
Jung-Fa Tsai ${ }^{1}$, Ming-Hua Lin ${ }^{2, *}$ and Duan-Yi Wen ${ }^{1}$ \\ 1 Department of Business Management, National Taipei University of Technology, Taipei 10608, Taiwan; \\ jftsai@ntut.edu.tw (J.-F.T.); t103749018@ntut.edu.tw (D.-Y.W.) \\ 2 Department of Urban Industrial Management and Marketing, University of Taipei, Taipei 11153, Taiwan \\ * Correspondence: mhlin@utaipei.edu.tw
}

Received: 24 August 2020; Accepted: 12 September 2020; Published: 16 September 2020

check for updates

\begin{abstract}
Several structural design problems that involve continuous and discrete variables are very challenging because of the combinatorial and non-convex characteristics of the problems. Although the deterministic optimization approach theoretically guarantees to find the global optimum, it usually leads to a significant burden in computational time. This article studies the deterministic approach for globally solving mixed-discrete structural optimization problems. An improved method that symmetrically reduces the number of constraints for linearly expressing signomial terms with pure discrete variables is applied to significantly enhance the computational efficiency of obtaining the exact global optimum of the mixed-discrete structural design problem. Numerical experiments of solving the stepped cantilever beam design problem and the pressure vessel design problem are conducted to show the efficiency and effectiveness of the presented approach. Compared with existing methods, this study introduces fewer convex terms and constraints for transforming the mixed-discrete structural problem and uses much less computational time for solving the reformulated problem to global optimality.
\end{abstract}

Keywords: global optimization; mixed-integer programming; mathematical methods; linearization; mixed-discrete variable; structural design problem

\section{Introduction}

Various problems that contain continuous and discrete variables arise in practical structural design applications; therefore, the mixed-discrete structural optimization (MDSO) problems have attracted increasing attention from researchers and practitioners in the last few decades. Some of the approaches for solving the MDSO problems belong to metaheuristics. Although the metaheuristic methods, such as tabu search, simulated annealing, evolutionary algorithms, and ant colony optimization, are robust and can successfully solve various types of problems [1], they cannot guarantee the global optimality of the obtained solution.

A signomial geometric function can be expressed as $\sum_{i=1}^{k} c_{i}\left(\prod_{j}^{n} x_{j}^{\alpha_{i j}}\right)$, where $c_{i}, \alpha_{i j} \in R$. Signomial geometric functions occur frequently in many engineering optimization problems such as chemical equilibrium problems [2], structural mechanics [3], integrated circuit design [4], stochastic processes [5], and aircraft design [6]. Since many nonlinear programming problems can be constructed or restated as signomial geometric programming problems by a simple change of variables or by an algebraic manipulation of terms [7], developing a global optimization approach for signomial geometric programming problems is useful for treating practical applications. The focus of this article is on the deterministic optimization approach for globally solving the MDSO problems that can be formulated as signomial geometric programming problems with mixed-discrete variables, such as the stepped cantilever beam design problem, the spring design problem, and the pressure vessel design problem. 
The engineering optimization problems that contain signomial geometric functions are NP-hard and difficult to be solved due to the combinatorial and non-convex characteristics of the problems. One major deterministic approach for globally solving the MDSO problems with signomial geometric functions is to transform the original problems as convex mixed-integer nonlinear programming (MINLP) problems. Some research [8-12] proposed general methods to treat generalized geometric problems and applied their methods to solve engineering design problems. Tsai and Lin [13], Lundell et al. [14], $\mathrm{Li}$ and $\mathrm{Lu}$ [15], and Lin et al. [16] solved generalized geometric problems or signomial geometric problems with continuous and discrete variables by using convexification and piecewise linearization techniques. Lin and Tsai [17] provided a complete survey of research on optimization approaches for the MDSO problems with signomial geometric functions, and proposed a deterministic method to solve the MDSO problems with signomial geometric functions by using convex transformations, linearization methods, and range reduction mechanisms. For all non-convex signomial geometric functions, Lin and Tsai [17] employed variable transformations to convexify all non-convex signomial terms, and then linearly approximated the inverse transformations. Experimental results indicated that their approach obtained an approximately globally optimal solution of the MDSO problem with a lower error in constraint compared with heuristic methods.

For treating pure discrete signomial terms in the MDSO problems, Tsai and Lin [18] proposed a linear transformation by using a logarithmic number of extra binary variables and constraints. Lu [19] developed a logarithmic method for globally solving the problems with the product of free-sign discrete functions. Both Tsai and Lin [18] and Lu [19] extended the Vielma and Nemhauser [20] method of dealing with special ordered set of type 1 (SOS1) constraints to linearize pure discrete signomial terms. Recently, Lin et al. [21] proposed a novel method based on the Li et al. [16] method to further decrease the constraints required in linearizing the discrete signomial term and efficiently treated the three-dimensional open-dimension rectangular packing problems. Tsai et al. [22] applied the $\mathrm{Li}$ et al. [23] method to solve the engineering design problems with discrete signomial terms. Lu [24] also proposed a similar approach for solving the product of free-sign discrete functions.

Although the deterministic optimization approach provides a theoretical guarantee of finding the global optimum, it usually leads to a significant burden in computational time. This study improves the reformulation method in Lin and Tsai [17] for the MDSO problems to enhance computational efficiency. Compared with the Lin and Tsai [17] method for the MDSO problems, this study has the following merits:

- Reduces the number of convex terms generated in reformulating the MDSO problems: Compared with the Lin and Tsai [17] method that converts the pure discrete signomial terms to convex terms and then linearizes the inverse transformation functions, this study directly linearizes the pure discrete signomial terms. Therefore, fewer convex terms are introduced in the transformed model;

- Decreases the number of binary variables and constraints required to linearize the inverse transformation functions of discrete variables: Compared with the Lin and Tsai [17] method, this study efficiently linearizes the inverse transformation functions of discrete variables by fewer binary variables and constraints.

The rest of the article is organized as follows. Section 2 describes the transformation techniques for convexifying a signomial term with pure discrete variables in the MDSO problems. Section 3 introduces an improved deterministic global optimization method for the MDSO problems. In Section 4, the stepped cantilever beam design problem and the pressure vessel design problem are efficiently solved to show the efficiency and effectiveness of the proposed method. After that, concluding remarks are made in Section 5.

\section{Global Optimization Techniques}

The mathematical programming model of an MDSO problem considered in this study, referring to Lin and Tsai [17], is expressed as follows: 
MDSO:

$$
\min f(\mathbf{x})=\sum_{p=1}^{T_{0}} c_{p} x_{1}^{\alpha_{p 1}} x_{2}^{\alpha_{p 2}} \cdots x_{n}^{\alpha_{p n}}
$$

subject to:

$$
\begin{gathered}
g_{k}(\mathbf{x}) \leq l_{k}, k=1, \cdots, K, \\
g_{k}(\mathbf{x})=\sum_{q=1}^{T_{k}} h_{k q} x_{1}^{\beta_{k q 1}} x_{2}^{\beta_{k q 2}} \cdots x_{n}^{\beta_{k q n}}, \\
\mathbf{x}=\left(x_{1}, x_{2}, \cdots, x_{n}\right), \underline{x}_{i} \leq x_{i} \leq \bar{x}_{i},
\end{gathered}
$$

where $x_{i} \in R, x_{i}>0$ for $1 \leq i \leq n, x_{i}(1 \leq i \leq m)$ are integer/discrete variables, $x_{i}(m+1 \leq i \leq n)$ are continuous variables, $c_{p}, h_{k q}, l_{k}, \alpha_{p i}, \beta_{k q i} \in R, T_{k}, k=1, \cdots, K$, represent the numbers of monomial terms in the constraints, and $\underline{x}_{i}$ and $\bar{x}_{i}$ are the lower and upper bounds of the discrete variable $x_{i}$, respectively. $f(\mathbf{x})$ and $g_{k}(\mathbf{x})$ are mixed-discrete signomial functions.

Lin and Tsai [17] converted all non-convex signomial terms to convex terms in an MDSO problem and then linearly expressed the inverse transformation functions. The reformulated program can be solved by the conventional convex MINLP techniques to reach a global optimum. However, their method introduces many convex terms and linearizes many inverse transformation functions. Consider a pure discrete signomial term containing only integer/discrete variables $x_{i}(1 \leq i \leq m)$ as below:

$$
c \prod_{i=1}^{m} x_{i}^{\alpha_{i}}, \text { where } x_{i} \in\left\{d_{i, 1}, d_{i, 2}, \cdots, d_{i, r_{i}}\right\}, i=1,2, \cdots, m .
$$

This study uses an efficient variable substitution method to directly linearize the pure discrete signomial term without introducing additional convex terms. Li et al. [23] proposed an expression of binary vectors by fewer constraints compared to the Vielma and Nemhauser [20] method. Li et al. [23] symmetrically reduced the number of constraints by half from examining binary variables in the inequality constraints. This paper applies the Li et al. [23] method to symmetrically reduce the number of constraints in linearizing a pure discrete signomial term. Assume $r$ possible values exist for a discrete variable $x, x_{i} \in\left\{d_{1}, d_{2}, \cdots, d_{r}\right\}$. Each $j, 1 \leq j \leq r$, can be expressed as:

$$
j=1+\sum_{t=1}^{\theta} 2^{t-1} v_{t}, \forall v_{t} \in\{0,1\}, \quad \theta=\left\lceil\log _{2} r\right\rceil .
$$

Let $B(j)=\left(v_{1}, v_{2}, \cdots, v_{\theta}\right)$ be a binary vector of $j$ where entry $t$ of $B(j)$ denotes the value of $v_{t}$ in the above expression of $j, \forall j \in\{1, \cdots, r\}$. Some notations are introduced below:

$S^{+}(t)$ : the set composed of all $j$ having $v_{t}=1$ in $B(j)$, i.e., $S^{+}(t)=\left\{j \mid v_{t}=1\right.$ in $\left.B(j)\right\}$.

$S^{-}(t)$ : the set composed of all $j$ having $v_{t}=0$ in $B(j)$, i.e., $S^{-}(t)=\left\{j \mid v_{t}=0\right.$ in $\left.B(j)\right\}$.

The basis of Li et al.'s [23] logarithmic method is stated below.

Theorem 1. [23]: For an integer $r>0$, let $h=\left\lceil\log _{2} r\right\rceil$. A vector $\lambda=\left(\lambda_{1}, \cdots, \lambda_{r}\right)$ with $\lambda_{j} \geq 0$ and $\sum_{j=1}^{r} \lambda_{j}=1$ is a binary vector, if there is a binary vector $\boldsymbol{u}=\left(u_{1}, \cdots, u_{h}\right)$ satisfying the following linear system:

$$
\sum_{j \in S^{+}(t)} \lambda_{j}=u_{t} \text { for } t=1,2, \cdots, h,
$$

where $\lambda_{j} \geq 0, u_{t} \in\{0,1\}$ and $S^{+}(t)$ is defined as before.

In Theorem $1, \lambda_{j}$ are real numbers, but the equality constraints enforce $\lambda_{j}$ to be 0 or 1 . Lin et al. [21] extended the Li et al. [23] method for linearizing a pure discrete signomial term $\sum_{i=1}^{m} x_{i}^{\alpha_{i}}$ where $x_{i} \in\left\{d_{i, 1}, d_{i, 2}, \cdots, d_{i, r_{i}}\right\}, i=1,2, \cdots, m$. The following corollary introduced by Lin et al. [21] linearizes a signomial term with pure discrete variables: 
Corollary 1. [21]: Denote $z_{1}=f_{1}\left(x_{1}\right), z_{i}=z_{i-1} f_{i}\left(x_{i}\right)$ for $i=2, \cdots, m$, and $z_{m}=\prod_{i=1}^{m} f_{i}\left(x_{i}\right)$ where $f_{i}\left(x_{i}\right)>0, x_{i}$ are discrete variables, $x_{i} \in\left\{d_{i, 1}, d_{i, 2}, \cdots, d_{i, r_{i}}\right\}, i=1,2, \cdots, m$. The terms $z_{i}, i=1,2, \cdots, m$, can be linearly expressed by the following expressions:

$$
\begin{gathered}
z_{1}=\sum_{j=1}^{r_{1}} f_{1}\left(d_{1, j}\right) \lambda_{1, j}, \\
z_{2}=\sum_{j=1}^{r_{2}} f_{2}\left(d_{2, j}\right) \sigma_{2, j}, \sum_{j=1}^{r_{2}} \sigma_{2, j}=z_{1}, \\
z_{3}=\sum_{j=1}^{r_{3}} f_{3}\left(d_{3, j}\right) \sigma_{3, j}, \sum_{j=1}^{r_{3}} \sigma_{3, j}=z_{2}, \\
z_{m}=\sum_{j=1}^{r_{m}} f_{m}\left(d_{m, j}\right) \sigma_{m, j}, \sum_{j=1}^{r_{m}} \sigma_{m, j}=z_{m-1}, \\
z_{i-1}-\bar{z}_{i-1}\left(1-u_{i, t}\right) \leq \sum_{j \in S^{+}(t)} \sigma_{i, j} \leq z_{i-1}, \sum_{j \in S^{+}(t)} \sigma_{i, j} \leq \bar{z}_{i-1} u_{i, t}, \\
t=1, \ldots,\left[\log _{2} r_{i}\right], i=2,3, \cdots, m, \\
x_{i}=\sum_{j=1}^{r_{i}} d_{i, j} \lambda_{i, j}, \sum_{j=1}^{r_{i}} \lambda_{i, j}=1, i=1,2, \cdots, m, \sum_{j \in S^{+}(t)} \lambda_{i, j}=u_{i, t}, \\
t=1, \ldots,\left[\log _{2} r_{i}\right], i=1,2, \cdots, m,
\end{gathered}
$$

where $\forall \lambda_{i, j}, \sigma_{i, j} \geq 0, \forall u_{i, t} \in\{0,1\}$, and $\bar{z}_{i-1}$ are upper bounds of $z_{i-1}$ for $i=2,3, \cdots, m$.

According to Corollary $1, \sum_{j=1}^{m}\left\lceil\log _{2} r_{i}\right\rceil$ binary variables, $4 m-1+\sum_{i=1}^{m}\left\lceil\log _{2} r_{i}\right\rceil+3 \sum_{i=2}^{m}\left\lceil\log _{2} r_{i}\right\rceil$ constraints, and $\sum_{i=1}^{m}\left(r_{i}+1\right)+\sum_{i=2}^{m} r_{i}$ continuous variables are required to linearly express discrete variables $x_{i}$ and signomial $\prod_{i=1}^{m} x_{i}^{\alpha_{i}}$ where $x_{i} \in\left\{d_{i, 1}, d_{i, 2}, \cdots, d_{i, r_{i}}\right\}, i=1,2, \cdots, m[21]$.

This study linearizes the pure discrete signomial terms by the method of Theorem 1 . For the non-convex signomial terms containing at least one continuous variable, the following transformation techniques were used to convexify the non-convex terms. Assume a positive mixed-discrete signomial term expressed as below:

$$
c \prod_{i=1}^{n} x_{i}^{\alpha_{i}}, \text { where } c>0, x_{i}>0, x_{i} \text { are continuous or discrete variables. }
$$

This positive non-convex term can be converted into a convex term $c \prod_{i \in I} x_{i}^{\alpha_{i}} e^{\sum_{j \neq I} \alpha_{j} y_{j}}$ where $I=\left\{k \mid \alpha_{p}<0, p=1,2, \cdots, n\right\} \cdot y_{j}=L\left(\ln x_{j}\right)$ is a linear function of the inverse transformation $\ln x_{j}$ [17]. In this study, if $x_{j}$ is a discrete variable, $x_{j} \in\left\{d_{j, 1}, d_{j, 2}, \cdots, d_{j, r_{j}}\right\}$, then $L\left(\ln x_{j}\right)$ can be expressed as:

$$
\begin{gathered}
L\left(\ln x_{j}\right)=\sum_{k=1}^{r_{j}}\left(\ln d_{j, k}\right) \lambda_{k}, x_{j}=\sum_{k=1}^{r_{j}} d_{j, k} \lambda_{k}, \sum_{k=1}^{r_{j}} \lambda_{k}=1 \sum_{j \in S^{+}(t)} \lambda_{k}=u_{t}, \text { where } \lambda_{k} \geq 0, \\
u_{t} \in\{0,1\} \text {, and } S^{+}(t) \text { is defined as before. }
\end{gathered}
$$

Assume a negative mixed-discrete signomial term expressed as below:

$$
c \prod_{i=1}^{n} x_{i}^{\alpha_{i}} \text {, where } c<0, x_{i}>0, x_{i} \text { are continuous or discrete variables. }
$$

This negative non-convex term can be converted into a convex term $c \prod_{i \in I} x_{i}^{\alpha_{i}} \prod_{j \notin I} y_{j}^{\beta}, \beta=$ $\left(1-\sum_{i=1}^{\mathrm{w}} \alpha_{i}\right) /(n-w)$, where $0 \leq \alpha_{1} \leq \alpha_{2} \leq \cdots \leq \alpha_{p}, 0 \geq \alpha_{p+1} \geq \alpha_{p+2} \geq \cdots \geq \alpha_{n}$, and $\sum_{i=1}^{\mathrm{r}} \alpha_{i}<1$ for some largest integer $w$, such that $w \leq p, I=\{k \mid k=1,2, \cdots, w\} . y_{j}=L\left(x_{j}^{\alpha_{j} / \beta}\right)$ is a linear function of the inverse transformation $x_{j}^{\alpha_{j} / \beta}$ [17].

Similarly, constraint (15) is used to linearize $x_{j}^{\alpha_{j} / \beta}$ if $x_{j}$ is a discrete variable. Compared to the Lin and Tsai [17] method, this study reduces the convex terms and eliminates the redundant constraints in equivalently reformulating the original MDSO problems into a convex MINLP problem. Since the 
method of Theorem 1 was sharp and locally ideal [21,23], the constructed model was as tight as the model constructed by Lin and Tsai [17].

\section{Global Optimization Approach for the MDSO Problems}

Figure 1 indicates the solving process of the proposed deterministic approach. First, the pure discrete signomial terms are directly linearized by the transformation techniques described in Corollary 1. The non-convex signomial terms containing at least one continuous variable were transformed into convex terms by the convexification strategies for the positive or negative mixed-discrete signomial term described in Section 2. Then the inverse transformation functions of discrete variables were linearly expressed by the method described in Corollary 1 and the inverse transformation functions of continuous variables were linearly approximated on the variable bounds with $m$ line segments.

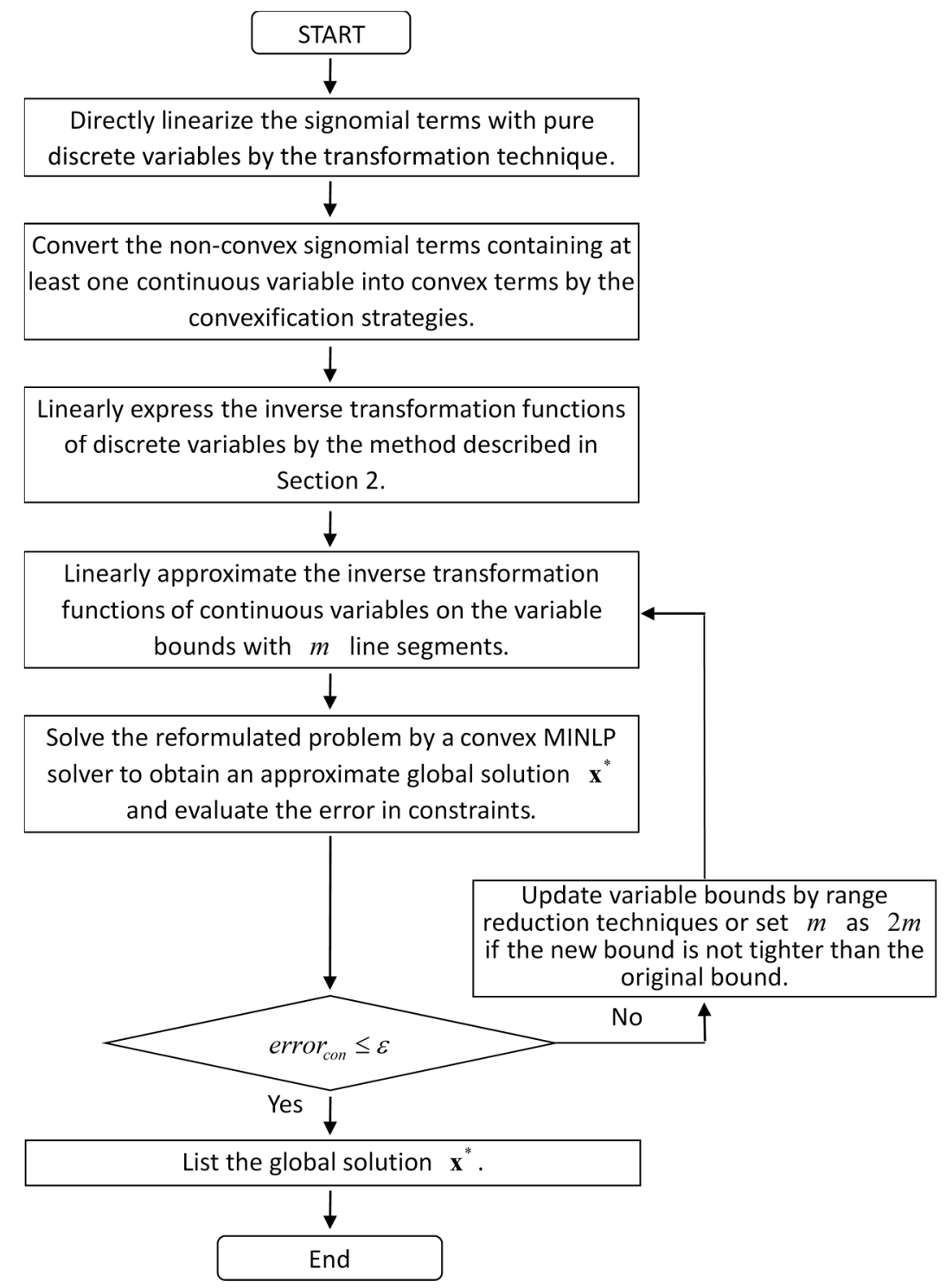

Figure 1. Global optimization approach for the mixed-discrete structural optimization (MDSO) problems.

After the original problem was transformed into a convex MINLP problem, a convex MINLP solver was used to solve the reformulated problem for obtaining an approximate global solution $\mathbf{x}^{*}$ and to evaluate the error in constraints error $_{\text {con }}=\max _{k}\left(g_{k}\left(\mathbf{x}^{*}\right)-l_{k}, 0\right)$. If error $_{\text {con }} \leq \varepsilon$, then list the global solution $\mathbf{x}^{*}$. Otherwise, update the variable range by the range reduction method [17]. 
If the new variable bound was tighter than the original variable bound, then remain using $m$ line segments to linearly approximate the inverse transformations of continuous variables on the new variable bound. Otherwise, set $m$ as $2 m$ to use more line segments in piecewise linearization for reducing the approximation error on the original variable bound.

\section{Numerical Examples}

The purpose of the numerical experiments described below is to demonstrate the enhancements in computational efficiency of the proposed method for the MDSO problems compared with current methods. All reformulated models were solved on a PC with a $3.2 \mathrm{GHz}$ Intel Core i5-4570 CPU and 8 GB memory (Santa Clara, CA, USA).

Example 1. This structural design problem minimizes the volume of the beam drawn from Erbatur et al. [25]. Figure 2 illustrates the structure of a five-stepped cantilever beam with a rectangular shape. Ten variables are used in this problem. $H_{i}$ and $B_{i}$ represent the heights and widths, respectively, in all five steps of the cantilever beam. $x_{1}\left(B_{4}\right), x_{2}\left(H_{4}\right), x_{3}\left(B_{5}\right)$, and $x_{4}\left(H_{5}\right)$ are continuous variables, $x_{5}\left(B_{1}\right)$ and $x_{6}\left(H_{1}\right)$ are integers, and $x_{7}\left(B_{2}\right)$, $x_{8}\left(H_{2}\right), x_{9}\left(B_{3}\right)$, and $x_{10}\left(H_{3}\right)$ are discrete variables. The mathematical programming model of the structural design problem can be formulated as follows:

$$
\min f(\mathbf{x})=100\left(x_{5} x_{6}+x_{7} x_{8}+x_{9} x_{10}+x_{1} x_{2}+x_{3} x_{4}\right),
$$

subject to:

$$
\begin{aligned}
& g_{1}(\mathbf{x})=10.7143-\frac{x_{5} x_{6}^{2}}{10^{3}} \leq 0, \\
& g_{2}(\mathbf{x})=8.5714-\frac{x_{7} x_{8}^{2}}{10^{3}} \leq 0, \\
& g_{3}(\mathbf{x})=6.4286-\frac{x_{9} x_{10}^{2}}{10^{3}} \leq 0, \\
& g_{4}(\mathbf{x})=4.2857-\frac{x_{1} x_{2}^{2}}{10^{3}} \leq 0, \\
& g_{5}(\mathbf{x})=2.1429-\frac{x_{3} x_{4}^{2}}{10^{3}} \leq 0, \\
& g_{6}(\mathbf{x})=10^{4}\left(\frac{244}{x_{5} x_{6}^{3}}+\frac{148}{x_{7} x_{8}^{3}}+\frac{76}{x_{9} x_{10}^{3}}+\frac{28}{x_{1} x_{2}^{3}}+\frac{4}{x_{3} x_{4}^{3}}\right)-1086 \leq 0, \\
& g_{7}(\mathbf{x})=x_{6}-20 x_{5} \leq 0, \\
& g_{8}(\mathbf{x})=x_{8}-20 x_{7} \leq 0, \\
& g_{9}(\mathbf{x})=x_{10}-20 x_{9} \leq 0 \text {, } \\
& g_{10}(\mathbf{x})=x_{2}-20 x_{1} \leq 0, \\
& g_{11}(\mathbf{x})=x_{4}-20 x_{3} \leq 0, \\
& x_{1}, x_{3} \in[1,5], x_{2}, x_{4} \in[30,65], x_{5} \in(1,2, \cdots, 5), x_{6} \in(30,31, \cdots, 65) \text {, } \\
& x_{7}, x_{9} \in(2.4,2.6,2.8,3.1) \text {, and } x_{8}, x_{10} \in(45.0,50.0,55.0,60.0) \text {. }
\end{aligned}
$$



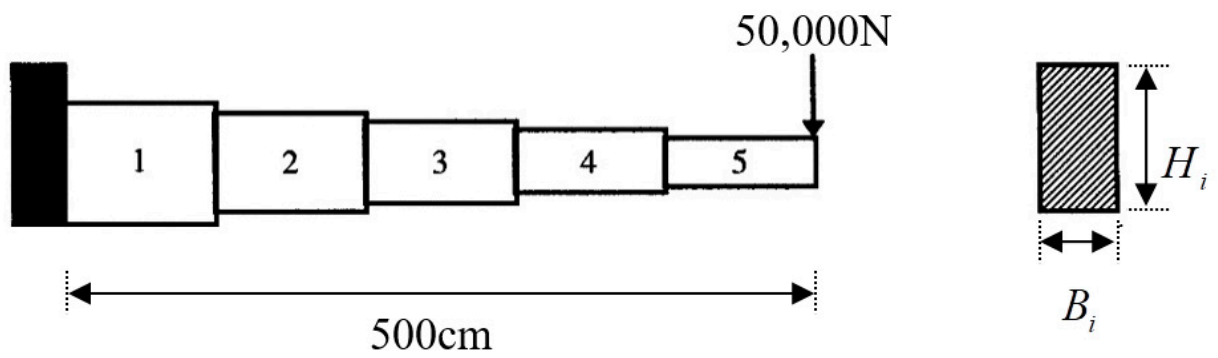

Figure 2. A stepped cantilever beam.

Six pure discrete signomial terms: $x_{5} x_{6}, x_{7} x_{8}, x_{9} x_{10},-x_{5} x_{6}^{2},-x_{7} x_{8}^{2},-x_{9} x_{10}^{2}$ exist in Example 1 . By the proposed method, these six discrete signomial terms are directly linearized. For the positive signomial terms containing continuous variables, $x_{1} x_{2}$ and $x_{3} x_{4}$ are convexified as $e^{z_{1}+z_{2}}$ and $e^{z_{3}+z_{4}}$, respectively. For the negative signomial terms containing continuous variables, $-x_{1} x_{2}^{2}$ and $-x_{3} x_{4}^{2}$ are convexified as $-y_{1}^{\frac{1}{3}} y_{2}^{\frac{2}{3}}$ and $-y_{3}^{\frac{1}{3}} y_{4}^{\frac{2}{3}}$, respectively. Then the inverse transformation functions are approximately linearized. This problem is equivalently reformulated as:

$$
\min f(\mathbf{x})=100\left(w_{1}+w_{2}+w_{3}+e^{z_{1}+z_{2}}+e^{z_{3}+z_{4}}\right),
$$

subject to:

$$
\begin{gathered}
g_{1}(\mathbf{x})=10.7143-\frac{w_{4}}{10^{3}} \leq 0, \\
g_{2}(\mathbf{x})=8.5714-\frac{w_{5}}{10^{3}} \leq 0, \\
g_{3}(\mathbf{x})=6.4286-\frac{w_{6}}{10^{3}} \leq 0, \\
g_{4}(\mathbf{x})=4.2857-\frac{y_{1}^{\frac{1}{3}} y_{2}^{\frac{2}{3}}}{10^{3}} \leq 0, \\
g_{5}(\mathbf{x})=2.1429-\frac{y_{3}^{\frac{1}{3}} y_{4}^{\frac{2}{3}}}{10^{3}} \leq 0, \\
g_{6}(\mathbf{x})=10^{4}\left(244 x_{5}^{-1} x_{6}^{-3}+148 x_{7}^{-1} x_{8}^{-3}+76 x_{9}^{-1} x_{10}^{-3}+28 x_{1}^{-1} x_{2}^{-3}+4 x_{3}^{-1} x_{4}^{-3}\right)-1086 \leq 0, \\
g_{7}(\mathbf{x})=x_{6}-20 x_{5} \leq 0, \\
g_{8}(\mathbf{x})=x_{8}-20 x_{7} \leq 0, \\
g_{9}(\mathbf{x})=x_{10}-20 x_{9} \leq 0, \\
g_{10}(\mathbf{x})=x_{2}-20 x_{1} \leq 0, \\
g_{11}(\mathbf{x})=x_{4}-20 x_{3} \leq 0, \\
z_{i}=L\left(\ln x_{i}\right), i=1,2,3,4, \\
y_{i}=L\left(x_{i}^{3}\right), i=1,2,3,4, \\
x_{1}, x_{3} \in[1,5], x_{2}, x_{4} \in[30,65], x_{5} \in(1,2, \cdots, 5), x_{6} \in(30,31, \cdots, 65), \\
x_{7}, x_{9} \in(2.4,2.6,2.8,3.1), \text { and } x_{8}, x_{10} \in(45.0,50.0,55.0,60.0) . \\
w_{4}=L\left(x_{5} x_{6}\right), w_{2}=L\left(x_{7} x_{8}\right), w_{3}=L\left(x_{9} x_{10}\right), \\
w_{5}=L\left(x_{7} x_{8}^{2}\right), w_{6}=L\left(x_{9} x_{10}^{2}\right),
\end{gathered}
$$


$L\left(x_{5} x_{6}\right), L\left(x_{7} x_{8}\right), L\left(x_{9} x_{10}\right), L\left(x_{5} x_{6}^{2}\right), L\left(x_{7} x_{8}^{2}\right)$, and $L\left(x_{9} x_{10}^{2}\right)$ are linear functions of $x_{5} x_{6}, x_{7} x_{8}, x_{9} x_{10}$, $x_{5} x_{6}^{2}, x_{7} x_{8}^{2}$, and $x_{9} x_{10}^{2}$, respectively, and can be directly linearized by the method introduced in Section 2 . $L\left(\ln x_{i}\right)$ and $L\left(x_{i}^{3}\right)$ are linear functions of $\ln x_{i}$ and $x_{i}^{3}$, respectively. After piecewise linearizing the inverse transformation functions of continuous variables, this program was transformed into a convex MINLP problem and the reformulated problem could be solved on LINGO [26]. Table 1 lists the solution, objective value, CPU time, and error in constraint $\left(\max _{k}\left(g_{k}\left(\mathbf{x}^{*}\right)-l_{k}, 0\right)\right)$ by Lin and Tsai [17] and the proposed method under 16, 32, 64, and 48 line segments in the linear approximation process on the original variable bounds. The objective value approximated closer to the exact global solution as the number of line segments increased. With the same number of line segments, the global solution obtained by the proposed method was identical to that obtained by the Lin and Tsai [17] method; however, the proposed method spent less CPU time solving the reformulated model. The difference in CPU time became larger as the number of line segments increased. With 128 line segments, the CPU time for solving the reformulated model by the Lin and Tsai [17] method was almost 3 h; however, the proposed method required only $22 \mathrm{~min}$. Table 2 displays the solution, objective value, accumulated CPU time, and error in constraint in each iteration for Example 1 by Lin and Tsai [17] and the proposed method using range reduction techniques. Sixteen line segments were used for linearly approximating the inverse transformation functions of continuous variables in each iteration. The accumulated CPU time included the CPU time of solving the reformulated models and updating variable bounds. An error in constraint below $10^{-5}$ could be reached after two iterations. The Lin and Tsai [17] method and the proposed method also found identical optimal solution that was closer to the exact globally optimal solution than other existing heuristic methods $[25,27,28]$. Compared with the data in Table 1 , the range reduction techniques significantly decreased the CPU time.

Table 1. Comparisons of experimental results of Example 1 by different methods.

\begin{tabular}{|c|c|c|c|c|c|}
\hline \multirow[b]{2}{*}{$m$} & \multirow[b]{2}{*}{ Solution $\left(x_{1}, x_{2}, x_{3}, x_{4}, x_{5}, x_{6}, x_{7}, x_{8}, x_{9}, x_{10}\right)$} & \multirow{2}{*}{$\begin{array}{l}\text { Objective } \\
\text { Value }\end{array}$} & \multicolumn{2}{|c|}{ CPU Time (hh:mm:ss) } & \multirow{2}{*}{$\begin{array}{l}\text { Error in } \\
\text { Constraint }\end{array}$} \\
\hline & & & Lin and Tsai [17] & $\begin{array}{c}\text { Proposed } \\
\text { Method }\end{array}$ & \\
\hline 16 & $\begin{array}{c}(2.202244,44.044890,1.748798,34.975960 \\
3.0,60.0,3.1,55.0,2.6,50.0)\end{array}$ & $63,850.641469$ & 00:02:32 & 00:00:59 & 0.013452 \\
\hline 32 & $\begin{array}{c}(2.203906,44.078113,1.749476,34.989529 \\
3.0,60.0,3.1,55.0,2.6,50.0)\end{array}$ & $63,880.915782$ & 00:07:14 & 00:02:21 & 0.003775 \\
\hline 64 & $\begin{array}{c}(2.204395,44.087898,1.749733,34.994651 \\
3.0,60.0,3.1,55.0,2.6,50.0)\end{array}$ & $63,890.838703$ & 00:35:19 & 00:06:35 & 0.000923 \\
\hline 128 & $\begin{array}{c}(2.204503,44.090051,1.749753,34.995065 \\
3.0,60.0,3.1,55.0,2.6,50.0)\end{array}$ & $63,892.602486$ & 02:57:18 & 00:21:36 & 0.000295 \\
\hline
\end{tabular}

Table 2. Comparisons of experimental results of Example 1 by different methods with range reduction.

\begin{tabular}{|c|c|c|c|c|c|}
\hline \multirow[b]{2}{*}{ Iteration } & \multirow[b]{2}{*}{ Solution $\left(x_{1}, x_{2}, x_{3}, x_{4}, x_{5}, x_{6}, x_{7}, x_{8}, x_{9}, x_{10}\right)$} & \multirow[b]{2}{*}{$\begin{array}{l}\text { Objective } \\
\text { Value }\end{array}$} & \multicolumn{2}{|c|}{ Accumulated CPU Time (hh:mm:ss) } & \multirow[b]{2}{*}{$\begin{array}{l}\text { Error in } \\
\text { Constraint }\end{array}$} \\
\hline & & & Lin and Tsai [17] & $\begin{array}{l}\text { Proposed } \\
\text { Method }\end{array}$ & \\
\hline 1 & $\begin{array}{c}(2.202244,44.044890,1.748798,34.975960 \\
3.0,60.0,3.1,55.0,2.6,50.0)\end{array}$ & $63,850.641953$ & 00:02:42 & 00:00:42 & 0.013452 \\
\hline 2 & $\begin{array}{c}(2.204553,44.091065,1.749769,34.995373, \\
3.0,60.0,3.1,55.0,2.6,50.0)\end{array}$ & $63,893.490593$ & 00:44:43 & 00:11:36 & 0.000005 \\
\hline
\end{tabular}

Example 2. The pressure vessel design problem that minimizes the total cost of materials, forming, and welding of the pressure vessel shown in Figure 3 was introduced by Sandgren [29]. Four design variables $T_{s}=x_{1}$, $T_{h}=x_{2}, R=x_{3}$, and $L=x_{4}$ are used to represent the shell thickness, the thickness of the head, the inner radius, and the length of the cylindrical section of the vessel, respectively. $T_{s}$ and $T_{h}$ are discrete values which are integer multiples of 0.0625 inches, $R$ and L are continuous. Lu [19] developed a logarithmic method for solving optimization problems containing the product of free-sign discrete functions by converting all variables into 
discrete variables. $x_{1}$ and $x_{2}$ are discrete variables with discreteness 0.0625 , and $x_{3}$ and $x_{4}$ are integer variables. Example 2 can be expressed as follows:

$$
\min f(\mathbf{x})=0.6224 x_{1} x_{3} x_{4}+1.7781 x_{2} x_{3}^{2}+3.1661 x_{1}^{2} x_{4}+19.84 x_{1}^{2} x_{3}
$$

subject to:

$$
\begin{gathered}
g_{1}(\mathbf{x})=0.0193 x_{3}-x_{1} \leq 0, \\
g_{2}(\mathbf{x})=0.00954 x_{3}-x_{2} \leq 0, \\
g_{3}(\mathbf{x})=1296000-\pi x_{3}^{2} x_{4}-\frac{4}{3} \pi x_{3}^{3} \leq 0, \\
g_{4}(\mathbf{x})=x_{4}-240 \leq 0, \\
x_{1}, x_{2} \in\{0.0625,0.125,0.1875, \cdots, 6.1875\},
\end{gathered}
$$

$10 \leq x_{3} \leq 200,10 \leq x_{4} \leq 200, x_{3}$ and $x_{4}$ are integer variables.

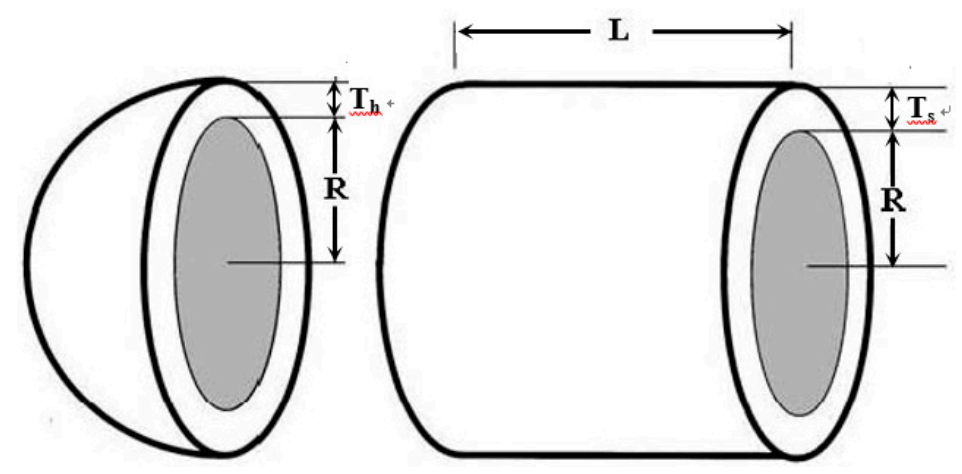

Figure 3. Pressure vessel design.

This problem can be linearly reformulated as follows:

$$
\min f(\mathbf{x})=0.6224 w_{1}+1.7781 w_{2}+3.1661 w_{3}+19.84 w_{4},
$$

subject to:

$$
\begin{gathered}
g_{1}(\mathbf{x})=0.0193 x_{3}-x_{1} \leq 0, \\
g_{2}(\mathbf{x})=0.00954 x_{3}-x_{2} \leq 0, \\
g_{3}(\mathbf{x})=1296000-\pi w_{5}-\frac{4}{3} \pi w_{6} \leq 0, \\
g_{4}(\mathbf{x})=x_{4}-240 \leq 0, \\
w_{1}=L\left(x_{1} x_{3} x_{4}\right), w_{2}=L\left(x_{2} x_{3}^{2}\right), w_{3}=L\left(x_{1}^{2} x_{4}\right), w_{4}=L\left(x_{1}^{2} x_{3}\right), \\
w_{5}=L\left(x_{3}^{2} x_{4}\right), w_{6}=L\left(x_{3}^{3}\right), \\
x_{1}, x_{2} \in\{0.0625,0.125,0.1875, \cdots, 6.1875\},
\end{gathered}
$$

$L\left(x_{1} x_{3} x_{4}\right), L\left(x_{2} x_{3}^{2}\right), L\left(x_{1}^{2} x_{4}\right), L\left(x_{1}^{2} x_{3}\right), L\left(x_{3}^{2} x_{4}\right)$, and $L\left(x_{3}^{3}\right)$ are linear expressions of $x_{1} x_{3} x_{4}, x_{2} x_{3}^{2}, x_{1}^{2} x_{4}$, $x_{1}^{2} x_{3}, x_{3}^{2} x_{4}$, and $x_{3}^{3}$, respectively, using the linearization techniques described in Section 2. Since all signomial terms involve only discrete variables, the transformed program was a mixed-integer linear programming problem solvable to reach a globally optimal solution. Table 3 displays the solution, 
objective value, and CPU time by Lin and Tsai [17] and the proposed method. The obtained global solution $(0.8125,0.4375,42,178)$ with objective 6074.99836 was the same by two methods. The proposed method required $10 \mathrm{~s}$; however, the Lin and Tsai [17] required $48 \mathrm{~s}$ to solve this problem.

Table 3. Experimental results of Example 2 by different methods.

\begin{tabular}{cccc}
\hline \multirow{2}{*}{ Solution $\left(x_{1}, x_{2}, x_{3}, x_{4}\right)$} & Objective Value & \multicolumn{2}{c}{ CPU Time (hh:mm:ss) } \\
\cline { 3 - 4 } & & Lin and Tsai [17] & Proposed Method \\
\hline$(0.8125,0.4375,42,178)$ & 6074.99836 & $00: 00: 48$ & $00: 00: 10$ \\
\hline
\end{tabular}

\section{Conclusions}

This study proposes a deterministic optimization approach for globally solving structural design problems formulated as signomial geometric programming problems with mixed-discrete variables. Efficient linearization techniques were used to directly linearize pure discrete signomial terms and to linearly express the inverse transformation functions of discrete variables, therefore resulting in a convex program with fewer convex terms and constraints. Experimental results indicated the proposed approach enhanced computational efficiency compared with current deterministic methods.

Author Contributions: Conceptualization, J.-F.T. and M.-H.L.; methodology, J.-F.T. and M.-H.L.; software, M.-H.L., and D.-Y.W.; validation, J.-F.T. and M.-H.L.; formal analysis, J.-F.T. and M.-H.L.; investigation, J.-F.T., M.-H.L., and D.-Y.W.; data curation, M.-H.L. and D.-Y.W.; writing-original draft preparation, J.-F.T. and M.-H.L.; writing-review and editing, J.-F.T. and M.-H.L.; visualization, M.-H.L. and D.-Y.W; supervision, J.-F.T and M.-H.L.; project administration, J.-F.T. and M.-H.L.; funding acquisition, J.-F.T. and M.-H.L. All authors have read and agreed to the published version of the manuscript.

Funding: This research was supported in part by the Ministry of Science and Technology in Taiwan under Grants MOST 108-2410-H-027-018, MOST 109-2410-H-027-012 -MY2 and MOST 108-2410-H-845-028.

Conflicts of Interest: The authors declare no conflict of interest.

\section{References}

1. Hedar, A. Studies on Metaheuristics for Continuous Global Optimization Problems Dissertation; Kyoto University: Kyoto, Japan, 2004.

2. Passy, U.; Wilde, D.J. A geometric programming algorithm for solving chemical equilibrium problems. SIAM J. Appl. Math. 1968, 16, 363-373. [CrossRef]

3. Ecker, J.G. Geometric programming: Methods, computations and applications. SIAM Rev. 1980, 22, 338-362. [CrossRef]

4. delM Hershenson, M.; Boyd, S.P.; Lee, T.H. Optimal design of a CMOS op-amp via geometric programming. IEEE 2001, 20,1-21. [CrossRef]

5. Feigin, P.D.; Passy, U. The geometric programming dual to the extinction probability problem in simple branching processes. Ann. Prob. 1981, 9, 498-503. [CrossRef]

6. Kirschen, P.G.; York, M.A.; Ozturk, B.; Hoburg, W.W. Application of signomial programming to aircraft design. J. Aircr. 2017, 55, 965-987. [CrossRef]

7. Shen, P.P.; Zhang, K.C. Global optimization of signomial geometric programming using linear relaxation. Appl. Math. Comput. 2004, 150, 99-114. [CrossRef]

8. Floudas, C.A.; Pardalos, P.M. State of the Art in Global Optimization: Computational Methods and Applications; Kluwer Academic Publisher: Boston, MA, USA, 1996.

9. Maranas, C.D.; Floudas, C.A. Global optimization in generalized geometric programming. Comput. Chem. Eng. 1997, 21, 351-369. [CrossRef]

10. Floudas, C.A. Global optimization in design and control of chemical process systems. J. Process. Control 1999, 10, 125-134. [CrossRef]

11. Floudas, C.A. Recent advances in global optimization for process synthesis, design and control: Enclosure of all solutions. Comput. Chem. Eng. 1999, 23, 963-974. [CrossRef] 
12. Floudas, C.A. Deterministic Global Optimization: Theory, Methods and Application; Kluwer Academic Publishers: Boston, CA, USA, 2000.

13. Tsai, J.F.; Lin, M.H. Global optimization of signomial mixed-integer nonlinear programming problems with free variables. J. Glob. Optim. 2008, 42, 39-49. [CrossRef]

14. Lundell, A.; Westerlund, J.; Westerlund, T. Some transformation techniques with applications in global optimization. J. Glob. Optim. 2009, 43, 391-405. [CrossRef]

15. Li, H.L.; Lu, H.C. Global optimization for generalized geometric programs with mixed free-sign variables. Oper. Res. 2009, 57, 701-713. [CrossRef]

16. Lin, M.H.; Tsai, J.F.; Wang, P.C. Solving engineering optimization problems by a deterministic global approach. Appl. Math. Inf. Sci. 2012, 6, 1101-1107.

17. Lin, M.H.; Tsai, J.F. A deterministic global approach for mixed-discrete structural optimization. Eng. Optim. 2014, 46, 863-879. [CrossRef]

18. Tsai, J.F.; Lin, M.H. An improved framework for solving NLIPs with signomial terms in the objective or constraints to global optimality. Comput. Chem. Eng. 2013, 53, 44-54. [CrossRef]

19. Lu, H.C. A logarithmic method for eliminating binary variables and constraints for the product of free-sign discrete function. Discret. Optim. 2013, 10, 11-24. [CrossRef]

20. Vielma, J.P.; Nemhauser, G. Modeling disjunctive constraints with a logarithmic number of binary variables and constraints. Math. Prog. 2011, 128, 49-72. [CrossRef]

21. Lin, M.H.; Tsai, J.F.; Chang, S.C. A superior linearization method for signomial discrete functions in solving three-dimensional open-dimension rectangular packing problems. Optimization 2017, 49, 746-761. [CrossRef]

22. Tsai, J.F.; Lin, M.H.; Peng, L.Y. Finding all global optima of engineering design problems with discrete signomial terms. Eng. Optim. 2020, 52, 165-184. [CrossRef]

23. Li, H.L.; Huang, Y.H.; Fang, S.C. A logarithmic method for reducing binary variables and inequality constraints in solving task assignment problems. INFORMS J. Comput. 2013, 25, 643-653. [CrossRef]

24. Lu, H.C. Improved logarithmic linearizing method for optimization problems with free-sign pure discrete signomial terms. J. Glob. Optim. 2017, 68, 95-123. [CrossRef]

25. Erbatur, F.; Hasancebi, O.; Tutuncu, I.; Kilic, H. Optimal design of planar and space structures with genetic algorithms. Comput. Struct. 2000, 75, 209-224. [CrossRef]

26. LINGO Release 17.0; LINDO System Inc.: Chicago, IL, USA, 2018.

27. Cai, J.; Thierauf, G. Evolution strategies for solving discrete optimization problems. Adv. Eng. Softw. 1996, 25, 177-183. [CrossRef]

28. Chen, T.Y.; Chen, H.C. Mixed-discrete structural optimization using a rank-niche evolution strategy. Eng. Optim. 2009, 41, 39-58. [CrossRef]

29. Sandgren, E. Nonlinear integer and discrete programming in mechanical design optimization. J. Mech. Des. 1990, 112, 223-229. [CrossRef]

(C) 2020 by the authors. Licensee MDPI, Basel, Switzerland. This article is an open access article distributed under the terms and conditions of the Creative Commons Attribution (CC BY) license (http://creativecommons.org/licenses/by/4.0/). 